〔ランチョンセミナー】

\title{
High-resolution screening of various biomarker compounds by using a new HPLC-Chip MS technology
}

R. Grimm Ph.D (Agilent Technologies Inc., Integrated Biology Solution Unit, Santa Clara, USA)

The discovery of biomarkers that are associated with particular diseases or disease progression is one of the key applications of proteomics research. For instance, it is well known that oligosaccharides are involved in a host of cell-cell processes and play a key role in the immune system. Changes in glycosylation or the formation of aberrant oligosaccharides accompany a host of diseases from infection to cancer (eg. ovarian cancer, diabetes, cystic fibrosis, arthritis, autoimmunity, respiratory capacity diseases). The same is true for proteins or endogenous peptides as well as for metabolic compounds.

In this presentation, we introduce a new fully automated and integrated analytical system for high-resolution profiling of different biomarker compound classes consisting of a chip-based chromatography system in conjunction with time-of-flight mass spectrometry. The microfluidic HPLC chips are made of laser ablated and laminated biocompatible polyimide films. Sample enrichment, separation and nanoelectrospray tips are fully integrated in the chip device. High-resolution separations of oligosaccharides are achieved with graphitized carbon column material packed in the chip device. Oligosaccharide isomers are readily separated and resolved. Specific oligosaccharide structures are identified by accurate mass measurements and highly reproducible retention times. In this way, over 100 different oligosaccharides are simultaneously identified and monitored. For the analysis of peptidic compounds we were deploying RP-18 packed chips that in combination with TOF-MS allowed us to separate more than 5000 compounds derived from isolated human serum glycoproteins (Zhang et al., Nature Biotech. 2003, 21, 660-666). 